Pak. j. sci. ind. res. Ser. B: biol. sci. 2020 63B(1) 22-29

\title{
Effect of Chelator EDTA on Phyto-Remediation of Cadmium, Chromium and Lead and their Effect on Growth of Sunflower (Helianthus annuus L.)
}

\author{
Jehan Bakht ${ }^{\mathrm{a} *}$, Rafi Ullah ${ }^{\mathrm{a}}$ and Mohammad Shafi ${ }^{\mathrm{b}}$ \\ anstitute of Biotechnology and Genetic Engineering, The University of Agriculture Peshawar, KPK, Pakistan \\ ${ }^{b}$ Department of Agronomy, The University of Agriculture Peshawar, KPK, Pakistan \\ (received January 26, 2018; revised July 15, 2018; accepted July 27, 2018)
}

\begin{abstract}
The present study investigates the phyto-accumulation capacity of two cultivars of sunflower (Helianthus annuus L.) for heavy metals. Analysis of the data recorded ten weeks after sowing indicated that heavy metal application had significantly $(\mathrm{p}<0.05)$ affected all the parameters under study. Interaction of EDTA $\times$ cultivar and EDTA $\times$ cultivar $\times$ heavy metal had significantly $(p<0.05)$ affected root fresh weight, root dry weight and heavy metal accumulation. EDTA application had significant $(p<0.05)$ effect on heavy metal accumulation when data was noted ten weeks after sowing. The data also revealed that maximum plant height, number of leaves/plant, shoot fresh weight and dry weight, root fresh and dry weight was noted in control pots $(0 \mathrm{mg} / \mathrm{kg}$ heavy metal). The suggested that maximum shoot fresh weight shoot dry weight, root fresh weight and root dry weight was noted in pots kept at control with San Sun-33 when applied with 5 mM EDTA. Similarly, maximum heavy metal accumulation was recorded in treatment sown of HiSun-33 applied with $5 \mathrm{mM}$ EDTA and $50 \mathrm{mM}$ chromium. Maximum root fresh weight was noted in control pots treated with 5 mM EDTA and planted with San Sun-33. Similarly, heavy metal accumulation was more in HiSun-33 treated with $50 \mathrm{mg} / \mathrm{kg}$ chromium and $5 \mathrm{mM}$ EDTA. In terms of accumulation of heavy metals, HiSun-33 demonstrated better accumulation of the tested heavy metals then SanSun-33, anyhow the growth of SanSun-33 was better than HiSun-33 due to lesser accumulation of heavy metals.
\end{abstract}

Keywords: phytoremediation, heavy metals, EDTA, sunflower

\section{Introduction}

Heavy metals are potential threat to the environment and human health. Heavy metals present in soil which can be taken in by plants specially edible plants and can enter into food chain. Heavy metals if accumulated are very harmful to health in lesser concentrations even in micromoles if accumulated (Michael et al., 2008). Various techniques including $e x$-situ treatment with physio-chemical and in-situ immobilization of heavy metal have been carried out to develop techniques for the remediation of contaminated soils which are generally costly and often harmful to different soil properties. Phyto-extraction of contaminated soils is a low cost method and is also environment friendly (Salt et al., 1998; Raskin et al., 1994; McGrath et al., 1993). The estimated cost of remediation heavy metals contaminated soils using conventional excavation, land filling etc. are approximately $\$ 150-350 /$ tonnes, while on the other hand, the estimated cost for phyto-remediation treatment of a lead polluted is $\$ 20$-50/tonnes. Several different plant species have been studied for their

*Author for correspondence; E-mail: jehanbakht@yahoo.co.uk potential application in removal of toxic wastes from the soils. Therefore, the use of plants to remove the contamination and clean up the hazardous waste is much better than the usual conventional methods.

Synthetic chelators are usually being used to increase the supply of micronutrients to plants in both soil and water. Similarly, use of chelator enhances phytoaccumulation by increasing heavy metals bioavailability thus enhancing uptake by plant and translocation of heavy metals from roots to the upper parts of the plants (Epstein et al., 1999). Ethylene diamine tetraacetic acid (EDTA) is one of the most effective agents to increase the accumulation of heavy metals in the aerial parts of plants (Hadi et al., 2014; Lipadzi et al., 2003).

\section{Materials and Methods}

The present study investigates the phyto-accomulation potential of sunflower cultivars (San Sun-33 and HiSun33) for $\mathrm{Pb}, \mathrm{Cr}$ and $\mathrm{Cd}$ uptake. Pots experiment using completely randomized (CR) design with three replications was used. Different heavy metals i.e. cadmium 
(10, 20 and $40 \mathrm{mg} / \mathrm{kg})$, chromium (50, 100 and $150 \mathrm{mg} / \mathrm{kg})$ and lead (100, 150 and $200 \mathrm{mg} / \mathrm{kg}$ ) were applied in the form of nitrate to the pots containing soil before sowing. EDTA was applied $(5 \mathrm{mg} / \mathrm{kg})$ to each treatment six weeks after sowing. Soil samples were collected before and after sowing for the determination of heavy metals concentration. Data was collected ten weeks after sowing on shoot fresh and dry weight, root fresh and dry weight, plant height and tissue heavy metal concentration. Standard agronomic practices were carried out during the experiment.

Statistical analysis. Data were analyzed statistically for analysis of variance (Gomez and Gomaz, 1984). MSTATC computer software was used for statistical analysis (Russel and Eisensmith, 1983). The significance of differences among means was determined by Least Significant Difference test (Steel and Torrie, 1997).

\section{Results and Discussion}

Plant growth and development. Heavy metal application had significantly $(\mathrm{p}<0.05)$ affected plant height, while the lone effect of cultivars and EDTA application and their interactions were non-significant $(\mathrm{p}>0.05)$. Taller plants were recorded by control treatments ( $0 \mathrm{mg} / \mathrm{kg}$ heavy metals) when compared with growth of the plant treated with cadmium, chromium and lead. Among the three heavy metals used, lead had more adverse effects on the growth of the plant than cadmium and chromium, when the plants were treated with $40 \mathrm{mg} / \mathrm{kg}$ cadmium, chromium and lead. The least adverse effect on plant height was demonstrated by plants treated with chromium. In case of cultivar $x$ EDTA application, maximum plant height was noted in San Sun-33 treated with 0 mM EDTA and minimum was observed in HiSun-33 when applied with $5 \mathrm{mM}$ EDTA. Among interaction between cultivars, heavy metals and EDTA application, maximum plant height was observed in treatments of San Sun-33 with 150 $\mathrm{mg} / \mathrm{kg}$ chromium and $0 \mathrm{mM}$ EDTA (Table 1). Minimum plant height was revealed by Hi sun-33 when treated with lead in the concentration of $150 \mathrm{mg} / \mathrm{kg}$. Babu et al. (2003) reported that the growth of L. gibba was significantly affected by the application of heavy metals.

Table 1. Plant height $(\mathrm{cm})$ of sunflower cultivars as affected by heavy metals and EDTA application ten weeks after sowing

\begin{tabular}{|c|c|c|c|c|c|c|}
\hline Varieties & EDTA & Heavy metals & Level-1 & Level-2 & Level-3 & $\begin{array}{l}\text { Variety } \times \text { EDTA } \times \\
\text { Heavy metals }\end{array}$ \\
\hline \multirow[t]{6}{*}{ HiSun-33 } & $0 \mathrm{mM}$ & Lead & 31.17 & 28.50 & 28.23 & 29.30 \\
\hline & $0 \mathrm{mM}$ & Chromium & 25.46 & 25.97 & 34.33 & 28.58 \\
\hline & $0 \mathrm{mM}$ & Cadmium & 34.73 & 36.23 & 31.83 & 34.26 \\
\hline & $5 \mathrm{mM}$ & Lead & 30.31 & 23.33 & 22.91 & 25.51 \\
\hline & $5 \mathrm{mM}$ & Chromium & 29.00 & 27.63 & 24.26 & 26.96 \\
\hline & $5 \mathrm{mM}$ & Cadmium & 25.01 & 32.17 & 28.87 & 28.68 \\
\hline \multirow[t]{6}{*}{ SanSun-33 } & $0 \mathrm{mM}$ & Lead & 53.67 & 48.50 & 54.00 & 2.05 \\
\hline & $0 \mathrm{mM}$ & Chromium & 51.33 & 49.33 & 47.50 & 49.38 \\
\hline & $0 \mathrm{mM}$ & Cadmium & 44.90 & 41.38 & 38.86 & 41.71 \\
\hline & $5 \mathrm{mM}$ & Lead & 44.38 & 48.73 & 48.46 & 47.19 \\
\hline & $5 \mathrm{mM}$ & Chromium & 50.97 & 43.5 & 38.97 & 44.48 \\
\hline & $5 \mathrm{mM}$ & Cadmium & 47.70 & 42.5 & 42.20 & 44.13 \\
\hline \multicolumn{7}{|l|}{ Variety $\times$ level } \\
\hline HiSun-33 & -- & -- & 29.28 & 28.97 & 28.40 & 28.88 \\
\hline SanSun-33 & -- & -- & 48.82 & 45.65 & 44.99 & 46.49 \\
\hline \multicolumn{7}{|l|}{ EDTA $\times$ levels } \\
\hline-- & $0 \mathrm{mM}$ & -- & 40.21 & 38.31 & 39.125 & 39.22 \\
\hline-- & $5 \mathrm{mM}$ & -- & 37.89 & 36.31 & 34.27 & 36.16 \\
\hline \multicolumn{7}{|c|}{ Heavy metals $\times$ levels } \\
\hline-- & -- & Lead & 39.88 & 37.26 & 38.40 & 38.51 \\
\hline-- & -- & Chromium & 39.19 & 36.60 & 36.26 & 37.35 \\
\hline-- & -- & Cadmium & 38.08 & 38.07 & 35.44 & 37.19 \\
\hline Control & -- & -- & 57.50 & 57.50 & 57.50 & 57.50 \\
\hline Rest (treatments) & -- & -- & 39.05 & 37.32 & 36.71 & 37.69 \\
\hline
\end{tabular}


Heavy metals had a significant $(\mathrm{p}<0.05)$ effect on number of leaves and the lone effect of cultivars and EDTA application and their interactions were nonsignificant. Regarding the production of leaves, more number of leaves/plant were recorded by plants kept at control ( $0 \mathrm{mg} / \mathrm{kg}$ heavy metals $)$ and minimum leaves production was noted in treatments of $150 \mathrm{mg} / \mathrm{kg}$ lead compared with other treatments. Number of leaves/plants was highest in treatment receiving $5 \mathrm{mM}$ EDTA. San Sun-33 recorded more leaves/plants applied with $5 \mathrm{mM}$ EDTA in case of interaction between cultivars and EDTA. Leaves/plant were more in control pots of San Sun-33 with or without EDTA and less in HiSun-33 treated with EDTA without the application of heavy metals (Table 2). Minimum number of leaves were counted in San Sun-33 when treated with lead in concentration of $150 \mathrm{mg} / \mathrm{kg}$ of soil and $5 \mathrm{mM}$ of EDTA, chromium and cadmium had moderate effects as compared to lead. These results are in agreement with
Vivek et al. (2000) who reported that Cd significantly decreased the growth and biomass of pea.

Heavy metal application had a significant $(\mathrm{p}<0.05)$ effect on shoot fresh weight, while the sole effect of cultivars and EDTA application and their possible interactions were non-significant. Shoot fresh weight was highest in pots treated with $0 \mathrm{mg} / \mathrm{kg}$ heavy metals (control) and minimum in pots treated with cadmium (40 mg/kg). Shoot fresh weight was more in treatment of 0 mM EDTA. San Sun-33 recorded maximum shoot fresh weight applied with $0 \mathrm{mM}$ EDTA. Maximum shoot fresh weight was produced in control pots of San Sun-33 with or without EDTA and minimum in San Sun-33 treated with $5 \mathrm{mM}$ EDTA and $150 \mathrm{mg} / \mathrm{kg}$ chromium (Table 3). These results are in agreement with Kastori et al. (1992) who reported that the accumulation of heavy metals had a significant effect on shoots and roots of the sunflower. Heavy metal

Table 2. Number of leaves/plant of sunflower cultivars as affected by heavy metals and EDTA application ten weeks after sowing

\begin{tabular}{|c|c|c|c|c|c|c|}
\hline Varieties & EDTA & Heavy metals & Level-1 & Level-2 & Level-3 & $\begin{array}{l}\text { Variety } \times \text { EDTA } \times \\
\text { heavy metals }\end{array}$ \\
\hline \multirow[t]{6}{*}{ HiSun-33 } & $0 \mathrm{mM}$ & Lead & 8.67 & 9.00 & 7.33 & 8.33 \\
\hline & $0 \mathrm{mM}$ & Chromium & 10.00 & 8.33 & 11.00 & 9.77 \\
\hline & $0 \mathrm{mM}$ & Cadmium & 10.00 & 10.67 & 10.00 & 10.2 \\
\hline & $5 \mathrm{mM}$ & Lead & 9.00 & 8.67 & 9.00 & 8.89 \\
\hline & $5 \mathrm{mM}$ & Chromium & 8.67 & 10.00 & 9.33 & 9.33 \\
\hline & $5 \mathrm{mM}$ & Cadmium & 9.33 & 10.33 & 9.00 & 9.55 \\
\hline \multirow[t]{6}{*}{ SanSun-33 } & $0 \mathrm{mM}$ & Lead & 11.33 & 10.33 & 10.67 & 10.77 \\
\hline & $0 \mathrm{mM}$ & Chromium & 11.33 & 9.67 & 11.67 & 10.89 \\
\hline & $0 \mathrm{mM}$ & Cadmium & 10.33 & 10.33 & 9.00 & 9.88 \\
\hline & $5 \mathrm{mM}$ & Lead & 9.33 & 11.67 & 10.67 & 10.55 \\
\hline & $5 \mathrm{mM}$ & Chromium & 11.67 & 12.00 & 10.00 & 11.22 \\
\hline & $5 \mathrm{mM}$ & Cadmium & 11.67 & 10.67 & 9.33 & 10.55 \\
\hline \multicolumn{7}{|l|}{ Variety $\times$ level } \\
\hline HiSun-33 & -- & -- & 9.27 & 9.50 & 9.27 & 9.35 \\
\hline SanSun-33 & -- & -- & 10.94 & 10.77 & 10.22 & 10.64 \\
\hline \multicolumn{7}{|l|}{ EDTA $\times$ levels } \\
\hline-- & $0 \mathrm{mM}$ & -- & 10.27 & 9.72 & 9.95 & 9.98 \\
\hline-- & $5 \mathrm{mM}$ & -- & 9.94 & 10.55 & 9.55 & 10.01 \\
\hline \multicolumn{7}{|c|}{ Heavy metals $\times$ levels } \\
\hline-- & -- & Lead & 9.58 & 9.91 & 9.42 & 9.63 \\
\hline-- & -- & Chromium & 10.41 & 10.00 & 10.50 & 10.30 \\
\hline-- & -- & Cadmium & 10.33 & 10.50 & 9.33 & 10.05 \\
\hline Control & -- & -- & 23.00 & 23.00 & 23.00 & 23.00 \\
\hline Rest (treatments) & -- & -- & 10.11 & 10.14 & 9.75 & 10 \\
\hline
\end{tabular}


Table 3. Shoot fresh weight $(\mathrm{g})$ of sunflower cultivars as affected by heavy metals and EDTA application ten weeks after sowing

\begin{tabular}{|c|c|c|c|c|c|c|}
\hline Varieties & EDTA & Heavy metals & Level-1 & Level-2 & Level-3 & $\begin{array}{l}\text { Variety } \times \text { EDTA } \times \\
\text { heavy metals }\end{array}$ \\
\hline \multirow[t]{6}{*}{ HiSun-33 } & $0 \mathrm{mM}$ & Lead & 19.17 & 17.67 & 13.30 & 16.71 \\
\hline & $0 \mathrm{mM}$ & Chromium & 14.71 & 15.54 & 17.07 & 15.77 \\
\hline & $0 \mathrm{mM}$ & Cadmium & 18.34 & 16.23 & 14.66 & 16.41 \\
\hline & $5 \mathrm{mM}$ & Lead & 17.97 & 10.65 & 14.24 & 14.28 \\
\hline & $5 \mathrm{mM}$ & Chromium & 10.87 & 14.08 & 13.89 & 12.94 \\
\hline & $5 \mathrm{mM}$ & Cadmium & 10.38 & 13.25 & 11.96 & 11.86 \\
\hline \multirow[t]{6}{*}{ SanSun-33 } & $0 \mathrm{mM}$ & Lead & 15.28 & 17.64 & 12.99 & 15.30 \\
\hline & $0 \mathrm{mM}$ & Chromium & 15.72 & 16.39 & 14.48 & 15.53 \\
\hline & $0 \mathrm{mM}$ & Cadmium & 10.65 & 15.15 & 10.19 & 11.99 \\
\hline & $5 \mathrm{mM}$ & Lead & 14.77 & 14.79 & 12.91 & 14.15 \\
\hline & $5 \mathrm{mM}$ & Chromium & 13.99 & 16.62 & 9.87 & 13.49 \\
\hline & $5 \mathrm{mM}$ & Cadmium & 16.10 & 12.36 & 12.44 & 13.63 \\
\hline \multicolumn{7}{|l|}{ Variety $\times$ level } \\
\hline HiSun-33 & -- & -- & 16.21 & 14.57 & 14.18 & 14.98 \\
\hline SanSun-33 & -- & -- & 13.84 & 15.49 & 12.14 & 13.82 \\
\hline \multicolumn{7}{|l|}{ EDTA $\times$ levels } \\
\hline-- & $0 \mathrm{mM}$ & -- & 15.20 & 16.43 & 13.78 & 15.14 \\
\hline-- & $5 \mathrm{mM}$ & -- & 14.68 & 13.625 & 12.55 & 13.62 \\
\hline \multicolumn{7}{|c|}{ Heavy Metals $\times$ levels } \\
\hline-- & -- & Lead & 15.57 & 15.18 & 13.36 & 14.70 \\
\hline-- & -- & Chromium & 13.82 & 15.65 & 13.82 & 14.43 \\
\hline-- & -- & Cadmium & 13.99 & 14.25 & 12.31 & 13.51 \\
\hline Control & -- & -- & 30.01 & 30.01 & 30.01 & 30.01 \\
\hline Rest (treatments) & -- & -- & 14.83 & 15.03 & 13.17 & 14.34 \\
\hline
\end{tabular}

application especially by lead had significantly $(\mathrm{p}<0.05)$ affected shoot dry weight and the lone effect of cultivars, EDTA application and interaction between cultivars $x$ EDTA $\times$ heavy metals application was non-significant. Shoot dry weight was maximum in pots treated with $0 \mathrm{mg} / \mathrm{kg}$ heavy metals (control) and minimum by treatments of lead $(100 \mathrm{mg} / \mathrm{kg})$. In case of EDTA application, shoot dry weight was more in pots treated with $5 \mathrm{mM}$ EDTA. Interaction between cultivars and EDTA application indicated that San Sun-33 produced more shoot dry weight when applied with $5 \mathrm{mM}$ EDTA. Shoot dry weight was more in control pots of San Sun-33 when applied with $5 \mathrm{mM}$ EDTA and minimum in San Sun-33 treated with $5 \mathrm{mM}$ EDTA and $100 \mathrm{mg} / \mathrm{kg}$ lead (Table 4). These results are in agreement with Stephen and Kochain (1998) who revealed that heavy metals accumulation reduced the root dry weights.

Heavy metal application and interaction between cultivars and EDTA had a significant $(\mathrm{p}<0.05)$ effect on root fresh weight and cultivars $\times$ EDTA $\times$ heavy metals application, while the sole effect of cultivars and EDTA application was non-significant. Root fresh weight was maximum in plants treated with $0 \mathrm{mg} / \mathrm{kg}$ heavy metals (control) and minimum in pots treated with chromium $(50 \mathrm{mg} / \mathrm{kg})$. These results are in agreement with Kastori et al. (1992) who reported that heavy metals application had more adverse effects on roots than shoots. Root fresh weight was highest in treatments of 5 mM EDTA. San Sun-33 produced maximum root fresh weight applied with $5 \mathrm{mM}$ EDTA. Root fresh weight was more in control pots of San Sun-33 when applied with $5 \mathrm{mM}$ EDTA and minimum was recorded in San Sun-33 treated with $5 \mathrm{mM}$ EDTA and $150 \mathrm{mg} / \mathrm{kg}$ lead in case of interaction among cultivars, heavy metals and EDTA application (Table 5). These results agree with Wong and Bradshaw (2006) who reported that application of heavy metals significantly affect the growth of roots in rye grass (Lolium perenne) and was toxic for the roots growth. Heavy metal application had a significant $(p<0.05)$ on root dry weight and the lone effect of cultivars and EDTA application and their all possible interactions were non-significant. Root dry weight was highest in pots treated with $0 \mathrm{mg} / \mathrm{kg}$ heavy metals (control) and minimum in pots treated with $20 \mathrm{mg} / \mathrm{kg}$ cadmium. Root dry weight was highest in treatments of $0 \mathrm{mM}$ EDTA. San Sun-33 recorded more root dry weight when applied with $0 \mathrm{mM}$ EDTA. Root dry weight was highest in control pots of San Sun-33 
Table 4. Shoot dry weight (g) of sunflower cultivars as affected by heavy metals and EDTA application ten weeks after sowing

\begin{tabular}{|c|c|c|c|c|c|c|}
\hline Varieties & EDTA & Heavy metals & Level-1 & Level-2 & Level-3 & $\begin{array}{l}\text { Variety } \times \text { EDTA } \times \\
\text { heavy metals }\end{array}$ \\
\hline \multirow[t]{6}{*}{ HiSun-33 } & $0 \mathrm{mM}$ & Lead & 1.97 & 2.07 & 1.54 & 1.86 \\
\hline & $0 \mathrm{mM}$ & Chromium & 1.95 & 1.71 & 2.47 & 2.04 \\
\hline & $0 \mathrm{mM}$ & Cadmium & 2.00 & 1.97 & 2.78 & 2.25 \\
\hline & $5 \mathrm{mM}$ & Lead & 2.33 & 0.98 & 1.03 & 1.44 \\
\hline & $5 \mathrm{mM}$ & Chromium & 1.54 & 1.58 & 2.81 & 1.97 \\
\hline & $5 \mathrm{mM}$ & Cadmium & 2.08 & 1.87 & 1.99 & 1.98 \\
\hline \multirow[t]{6}{*}{ SanSun-33 } & $0 \mathrm{mM}$ & Lead & 3.29 & 2.22 & 2.67 & 2.72 \\
\hline & $0 \mathrm{mM}$ & Chromium & 2.82 & 3.49 & 1.72 & 2.67 \\
\hline & $0 \mathrm{mM}$ & Cadmium & 1.79 & 2.27 & 1.57 & 1.87 \\
\hline & $5 \mathrm{mM}$ & Lead & 2.15 & 2.02 & 2.15 & 2.13 \\
\hline & $5 \mathrm{mM}$ & Chromium & 3.03 & 2.43 & 2.71 & 2.72 \\
\hline & $5 \mathrm{mM}$ & Cadmium & 1.61 & 2.59 & 1.29 & 1.83 \\
\hline \multicolumn{7}{|l|}{ Variety $\times$ level } \\
\hline HiSun-33 & & & 1.97 & 1.69 & 2.10 & 1.92 \\
\hline SanSun-33 & & & 2.44 & 2.50 & 2.01 & 2.32 \\
\hline \multicolumn{7}{|l|}{ EDTA $\times$ levels } \\
\hline $0 \mathrm{mM}$ & & & 2.30 & 2.28 & 2.15 & 2.23 \\
\hline $5 \mathrm{mM}$ & & & 2.12 & 1.91 & 1.99 & 2.01 \\
\hline \multicolumn{7}{|c|}{ Heavy metals $\times$ levels } \\
\hline & & Lead & 2.43 & 1.82 & 1.84 & 2.03 \\
\hline & & Chromium & 2.33 & 2.30 & 2.42 & 2.35 \\
\hline & & Cadmium & 1.87 & 2.17 & 1.90 & 1.98 \\
\hline Control & & & 4.66 & 4.66 & 4.66 & 4.66 \\
\hline Rest (treatments) & & & 2.21 & 2.10 & 2.06 & 2.12 \\
\hline
\end{tabular}

Table 5. Root fresh weight ( $\mathrm{g}$ ) of sunflower cultivars as affected by heavy metals and EDTA application ten weeks after sowing

\begin{tabular}{|c|c|c|c|c|c|c|}
\hline Varieties & EDTA & Heavy metals & Level-1 & Level-2 & Level-3 & $\begin{array}{l}\text { Variety } \times \text { EDTA } \times \\
\text { heavy metals }\end{array}$ \\
\hline \multirow[t]{6}{*}{ HiSun-33 } & $0 \mathrm{mM}$ & Lead & 1.99 & 2.31 & 1.85 & 2.05 \\
\hline & $0 \mathrm{mM}$ & Chromium & 1.76 & 2.06 & 2.41 & 2.07 \\
\hline & $0 \mathrm{mM}$ & Cadmium & 1.78 & 1.14 & 1.86 & 1.59 \\
\hline & $5 \mathrm{mM}$ & Lead & 1.54 & 1.79 & 4.63 & 2.65 \\
\hline & $5 \mathrm{mM}$ & Chromium & 1.43 & 1.74 & 2.53 & 1.90 \\
\hline & $5 \mathrm{mM}$ & Cadmium & 2.61 & 4.43 & 1.87 & 2.97 \\
\hline \multirow[t]{6}{*}{ SanSun-33 } & $0 \mathrm{mM}$ & Lead & 2.02 & 2.32 & 2.21 & 2.18 \\
\hline & $0 \mathrm{mM}$ & Chromium & 1.92 & 1.06 & 1.56 & 1.51 \\
\hline & $0 \mathrm{mM}$ & Cadmium & 1.29 & 1.88 & 1.94 & 1.70 \\
\hline & $5 \mathrm{mM}$ & Lead & 2.41 & 2.09 & 1.04 & 1.85 \\
\hline & $5 \mathrm{mM}$ & Chromium & 1.25 & 4.33 & 1.82 & 2.46 \\
\hline & $5 \mathrm{mM}$ & Cadmium & 1.98 & 2.31 & 2.43 & 2.24 \\
\hline \multicolumn{7}{|l|}{ Variety $\times$ level } \\
\hline HiSun-33 & -- & -- & 1.85 & 2.24 & 2.52 & 2.21 \\
\hline SanSun-33 & -- & -- & 1.81 & 2.33 & 1.83 & 1.99 \\
\hline \multicolumn{4}{|l|}{ EDTA $\times$ levels } & 1.79 & 1.97 & 1.85 \\
\hline & $5 \mathrm{mM}$ & -- & 1.87 & 2.78 & 2.38 & 2.34 \\
\hline \multicolumn{7}{|c|}{ Heavy metals $\times$ levels } \\
\hline-- & -- & Lead & 1.99 & 2.12 & 2.43 & 2.18 \\
\hline-- & -- & Chromium & 1.59 & 2.29 & 2.08 & 1.98 \\
\hline-- & -- & Cadmium & 1.91 & 2.44 & 2.02 & 2.12 \\
\hline Control & -- & -- & 4.75 & 4.75 & 4.75 & 4.75 \\
\hline Rest (treatments) & -- & -- & 1.83 & 2.28 & 2.17 & 2.09 \\
\hline
\end{tabular}


Table 6. Root dry weight (g) of Sunflower cultivars as affected by heavy metals and EDTA application ten weeks after sowing

\begin{tabular}{|c|c|c|c|c|c|c|}
\hline Varieties & EDTA & Heavy metals & Level-1 & Level-2 & Level-3 & $\begin{array}{l}\text { Variety } \times \text { EDTA } \times \\
\text { heavy metals }\end{array}$ \\
\hline \multirow[t]{6}{*}{ HiSun-33 } & $0 \mathrm{mM}$ & Lead & 0.17 & 0.43 & 0.39 & 0.33 \\
\hline & $0 \mathrm{mM}$ & Chromium & 0.81 & 0.74 & 0.19 & 0.58 \\
\hline & $0 \mathrm{mM}$ & Cadmium & 0.19 & 0.21 & 0.43 & 0.27 \\
\hline & $5 \mathrm{mM}$ & Lead & 0.82 & 0.29 & 0.46 & 0.52 \\
\hline & $5 \mathrm{mM}$ & Chromium & 0.42 & 0.39 & 0.72 & 0.51 \\
\hline & $5 \mathrm{mM}$ & Cadmium & 0.45 & 0.12 & 0.16 & 0.24 \\
\hline \multirow[t]{6}{*}{ SanSun-33 } & $0 \mathrm{mM}$ & Lead & 0.22 & 0.14 & 0.18 & 0.18 \\
\hline & $0 \mathrm{mM}$ & Chromium & 0.20 & 0.20 & 0.16 & 0.18 \\
\hline & $0 \mathrm{mM}$ & Cadmium & 0.09 & 0.13 & 0.11 & 0.11 \\
\hline & $5 \mathrm{mM}$ & Lead & 0.17 & 0.15 & 0.16 & 0.16 \\
\hline & $5 \mathrm{mM}$ & Chromium & 0.36 & 0.19 & 0.14 & 0.23 \\
\hline & $5 \mathrm{mM}$ & Cadmium & 0.12 & 0.16 & 0.18 & 0.15 \\
\hline \multicolumn{7}{|l|}{ Variety $\times$ level } \\
\hline HiSun-33 & -- & -- & 0.47 & 0.36 & 0.39 & 0.41 \\
\hline SanSun-33 & -- & -- & 0.19 & 0.16 & 0.15 & 0.17 \\
\hline \multicolumn{7}{|l|}{ EDTA $\times$ levels } \\
\hline-- & $0 \mathrm{mM}$ & -- & 0.28 & 0.30 & 0.24 & 0.27 \\
\hline-- & $5 \mathrm{mM}$ & -- & 0.39 & 0.21 & 0.30 & 0.30 \\
\hline \multicolumn{7}{|c|}{ Heavy metals $\times$ levels } \\
\hline -- & -- & Lead & 0.34 & 0.25 & 0.29 & 0.29 \\
\hline-- & -- & Chromium & 0.44 & 0.38 & 0.30 & 0.37 \\
\hline-- & -- & Cadmium & 0.21 & 0.15 & 0.22 & 0.19 \\
\hline Control & -- & -- & 1.75 & 1.75 & 1.75 & 1.75 \\
\hline Rest (treatments) & -- & -- & 0.33 & 0.26 & 0.27 & 0.29 \\
\hline
\end{tabular}

with 5 mM EDTA (Table 6). These results are in conformity with Anna-Maj (1989). He reported that heavy metals applications was toxic to small roots, inhibited its growth and lessen its biomass production.

Heavy metals accumulation. Heavy metal and EDTA application had significantly $(\mathrm{p}<0.05)$ affected heavy metal accumulation and non-significant $(p>0.05)$ effect of cultivars and interaction between cultivars and EDTA and cultivars $\times$ EDTA $\times$ heavy metals application was observed. The data suggested that heavy metal accumulation was highest in plants treated with 150 $\mathrm{mg} / \mathrm{kg}$ chromium while minimum was noted in $10 \mathrm{mg} /$ $\mathrm{kg}$ cadmium treated pots (Table 7 ). In case of EDTA application, heavy metals accumulation was increased when plants were applied with $5 \mathrm{mM}$ EDTA, especially accumulation of chromium was recorded greater than cadmium and lead (Table 7). These results are in agreement with Vivek et al. (2000) and Wong et al. (2006), who in their individual studies reported that EDTA acted as chelating agent for the accumulation of heavy metals. EDTA also act as chelating agent for nutrients other than heavy metals like potassium and calcium etc. (Shahandeh et al., 2002) anyhow some studies suggested that EDTA did not have any effect on the accumulation of heavy metals by plants. Interaction between cultivars and EDTA application showed that HiSun-33 accumulated maximum heavy metals when treated with $5 \mathrm{mM}$ EDTA and minimum by San Sun-33 treated with 0 mM EDTA. Heavy metal accumulation was more in HiSun-33 when applied with $5 \mathrm{mM}$ EDTA and $150 \mathrm{mg} / \mathrm{kg}$ chromium while minimum heavy metals (10 mg/kg cadmium) were accumulated by $\mathrm{Hi}$ Sun-33 when treated with $0 \mathrm{mM}$ EDTA (Table 7). These results are in agreement with Grcman et al. (2001), who reported that application of EDTA enhanced accumulation of heavy metals by plants and act as chelating agents. Andrew et al. (1998) suggested that EDTA significantly increases the accumulation of heavy metals in Indian mustard. These results also agree with (Michael et al., 2008; Liphadzi and Kirkhan, 2006; Shahandeh and Hossener, 2002; Jianwei et al., 1997), however, contradictory to Lipadzi et al. (2003) who reported that EDTA application did not affect the uptake of the applied heavy metals. 
Table 7. Heavy metals $(\mathrm{Pb}, \mathrm{Cr}$ and $\mathrm{Cd})$ accumulation by sunflower cultivars as affected by heavy metals and EDTA application ten weeks after sowing

\begin{tabular}{|c|c|c|c|c|c|c|}
\hline Varieties & EDTA & Heavy metals & Level-1 & Level-2 & Level-3 & $\begin{array}{l}\text { Variety } \times \text { EDTA } \times \\
\text { heavy metals }\end{array}$ \\
\hline \multirow{6}{*}{ HiSun-33 } & $0 \mathrm{mM}$ & Lead & 33.25 & 36.25 & 143.3 & 70.91 \\
\hline & $0 \mathrm{mM}$ & Chromium & 188.3 & 271.5 & 332.8 & 264.16 \\
\hline & $0 \mathrm{mM}$ & Cadmium & 1.93 & 2.1 & 8.23 & 4.08 \\
\hline & $5 \mathrm{mM}$ & Lead & 77.00 & 139.5 & 123.6 & 113.36 \\
\hline & $5 \mathrm{mM}$ & Chromium & 270.5 & 266 & 364.8 & 300.41 \\
\hline & $5 \mathrm{mM}$ & Cadmium & 3.43 & 26.98 & 27.4 & 19.27 \\
\hline \multirow[t]{6}{*}{ SanSun-33 } & $0 \mathrm{mM}$ & Lead & 37.88 & 56.13 & 69.88 & 54.63 \\
\hline & $0 \mathrm{mM}$ & Chromium & 161.5 & 186 & 271.6 & 206.39 \\
\hline & $0 \mathrm{mM}$ & Cadmium & 2.15 & 5.44 & 6.64 & 4.743 \\
\hline & $5 \mathrm{mM}$ & Lead & 71.75 & 145 & 143.1 & 119.96 \\
\hline & $5 \mathrm{mM}$ & Chromium & 248.5 & 188.5 & 319.4 & 252.12 \\
\hline \multirow{2}{*}{\multicolumn{7}{|c|}{ Variety $\times$ level }} \\
\hline & & & & & & \\
\hline HiSun-33 & -- & -- & 95.73 & 123.7 & 166.7 & 128.70 \\
\hline SanSun-33 & -- & -- & 88.04 & 99.32 & 138.1 & 108.49 \\
\hline \multicolumn{7}{|l|}{ EDTA $\times$ levels } \\
\hline-- & $0 \mathrm{mM}$ & -- & 70.83 & 92.9 & 138.7 & 100.82 \\
\hline-- & $5 \mathrm{mM}$ & -- & 112.9 & 130.1 & 166.1 & 136.37 \\
\hline \multicolumn{7}{|c|}{ Heavy Metals $\times$ levels } \\
\hline-- & -- & Lead & 54.97 & 94.22 & 120 & 89.72 \\
\hline-- & -- & Chromium & 217.2 & 228 & 322.1 & 255.7 \\
\hline-- & -- & Cadmium & 3.478 & 12.35 & 15.08 & 10.31 \\
\hline Control & -- & -- & 0.04 & 0.04 & 0.04 & 0.04 \\
\hline Rest (treatments) & -- & -- & 91.88 & 111.5 & 152.4 & 118.59 \\
\hline
\end{tabular}

Conflict of Interest. The authors declare that there is no conflict of interest.

\section{References}

Andrew, D.V., Kapulnik, Y., Raskin, I., Salt, D.E. 1998. The role of EDTA in lead transport and accumulation by Indian mustard. Plant Physiology, 117: 447-453.

Anna-Maj, B.P. 1989. Toxicity of heavy metals (Zn, $\mathrm{Cu}, \mathrm{Cd}, \mathrm{Pb}$ ) to vascular plants. Water Air and Soil Pollution, 47: 287-319.

Babu, T.S., Akthar, T.A., Lampi, M.A., Tripuranthakam, S., Dixon, D.G., Greengerg, B.M. 2003. Similar stress responses elicited by copper and ultraviolet radiation in the aquatic plant Lemna gibba: implication of reactive oxygen species as common signals. Plant Cell Physiology, 44: 1320-1329.

Epstein, A.L., Gussman, C.D., Blaylock, M.J., Yermiyahu, U., Huang, W.J., Kapulnik, Y., Orser, C.S. 1999. EDTA and Pb-EDTA accumulation in Brassica juncea grown in Pb-amended soil. Plant Soil, 208: 87-94.

Gomez, K.A., Gomez, A.A. 1984. Statistical Procedures for Agricultural Research. $2^{\text {nd }}$ eds. John Willey and
Sons Inc., NewYork, USA.

Grcman, H., Velikonja-Bolta, S., Vodnik, D., Kos, B, Leštan, D. 2001. EDTA enhanced heavy metal phyto-extraction: metal accumulation, leaching and toxicity. Plant and Soil, 235: 105-114.

Hadi, F., Ali, N., Ahmad, A. 2014. Enhanced phytoremediation of cadmium contaminated soil by Parthenium hysterophorus plant: effect of gibberellic acid (GA) and synthetic chelator, alone and in combinations. Bioremediation Journal, 18: 46-55. Jianwei, W.H., Chen, J., Berti, W.R., Cunningham, S.D. 1997. Phyto-remediation of lead-contaminated soils: role of synthetic chelates in lead phytoextraction. Environmental Science and Technology, 31: 800-805.

Kastori, R., Petrovic, M., Petroic, N. 1992. Effect of excess lead, cadmium, coppers and zinc on water relations in sunflower. Journal of Plant Nutrition, 15: 2427-2439.

Liphadzi, M.S., Kirkham, M.S. 2006. Chelate-assisted heavy metal removal by sunflower to improve soil with sludge. Journal of Crop Production, 16: 153172.

Liphadzi, M.S., Kirkham, M.B., Mankin, M.B., Paulsen, 
K.R. 2003. Role of chelating agents in removal of Heavy metals. Plant and Soil, 257: 171-182.

McGrath, S.P., Sidoli, C.M.D., Baker, A.J.M., Reeves, R.D. 1993. The potential for the use of metalaccumulating plants for the in situ decontamination of metal polluted soils. Integrated Soil and Sediment Research, 12: 673-677.

Michael, J.B., Salt, D.E., Dushenkov, S., Zakharova, O., Gussman, Kapulnik, Y., Ensley, B.D., Raskin, I. 2008. Enhanced accumulation of $\mathrm{Pb}$ in Indian mustard by soil applied chelating agents. Environmental Science and Technology, 31: 860-865.

Raskin, I., Kumar, P.B.A.N., Dushenkov, S., Salt, D.E. 1994. Bio-concentration of heavy metals by plants. Current Opinion in Biotechnology, 5: 285-290.

Russel, D.F., Eisensmith, S.P. 1983. MSTATC. Crop and Soil Science Department, Michigan State University, USA.

Salt, D.E., Smith, R.D., Raskin, I. 1998. Phyto-remediation. Annual Review of Plant Physiology and
Molecular Biology, 49: 643-668.

Shahandeh, H., Hossner, L.R. 2002. Enhancement of uranium phyto-accumulation from contaminated soils. Soil Science, 167: 269-280.

Steel, R.G.D., Torrie, J.H. 1997. Principles and Procedures of Statistics. McGraw Hill Book Company Inc., New York, USA.

Stephen, D.E., Kochian, L.V. 1998. Phyto-extraction of zinc by oat (Avena sativa), barley (Hordeum vulgare), and Indian mustard (Brassica juncea). Environmental Science and Technology, 32: 802806.

Vivek, D., Pandey, V., Shyam, R. 2000. Differential antioxidative responses to cadmium in roots and leaves of pea (Pisum sativum L. cv. Azad). Journal of Experimental Botany, 52: 1101-1109.

Wong, M.H., Bradshaw, A.D. 2006. A comparison of the toxicity of heavy metals using root elongation of rye grass, Lolium perenne. New Phytology, 10: 1469-8137. 